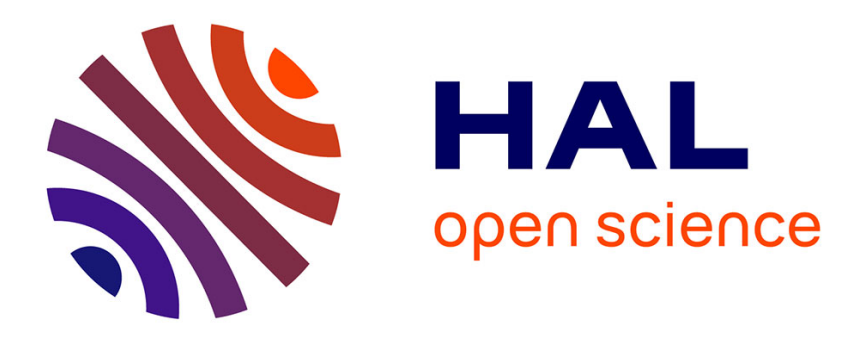

\title{
Particle-Particle Hole-Hole TDA - And Beyond - For The Nuclear Pairing Hamiltonian
}

\author{
H. Molique, J. Dudek
}

\section{To cite this version:}

H. Molique, J. Dudek. Particle-Particle Hole-Hole TDA - And Beyond - For The Nuclear Pairing Hamiltonian. 13th WORKSHOP ON NUCLEAR PHYSICS "MARIE AND PIERRE CURIE" PAIRING AND BEYOND - 50 YEARS OF THE BCS MODEL, Sep 2006, Kazimierz Dolny, Poland. pp.298-309, 10.1142/S0218301307005739 . in2p3-00135117

\section{HAL Id: in2p3-00135117 https://hal.in2p3.fr/in2p3-00135117}

Submitted on 6 Mar 2007

HAL is a multi-disciplinary open access archive for the deposit and dissemination of scientific research documents, whether they are published or not. The documents may come from teaching and research institutions in France or abroad, or from public or private research centers.
L'archive ouverte pluridisciplinaire HAL, est destinée au dépôt et à la diffusion de documents scientifiques de niveau recherche, publiés ou non, émanant des établissements d'enseignement et de recherche français ou étrangers, des laboratoires publics ou privés. 
October 25,

\title{
PARTICLE-PARTICLE HOLE-HOLE TDA - AND BEYOND - FOR THE NUCLEAR PAIRING HAMILTONIAN
}

\author{
Hervé MOLIQUE \\ IUFM d'Alsace and IPHC/IReS-ULP \\ 23, rue du Loess B.P. 28 - F-67037 Strasbourg Cedex 2, France \\ Herve.Molique@IReS.in2p3.fr \\ Jerzy DUDEK \\ IPHC/IReS-ULP \\ 23, rue du Loess B.P. 28 - F-67037 Strasbourg Cedex 2, France \\ Jerzy.Dudek@IReS.in2p3.fr \\ Received (received date) \\ Revised (revised date)
}

\begin{abstract}
A comparison of different seniority zero solutions to the picket-fence model for the nuclear pairing hamiltonian problem is performed. These solutions are calculated, in the normal regime, within the self-consistent Random Phase Approximation (SCRPA) and various simplifications of this formalism, and also with the Tamm-Dancoff approach in the particle-particle-hole-hole channel (pphh-TDA). The latter formalism represents a first approximation to the earlier developped so-called P-Symmetric Many-Body method (PSY-MB). In the superfluid regime, the solutions are compared with the BCS results. By comparing the results with the exact ones, obtained by the Richardson method, it is shown that the PSY-MB method provides a powefull tool in solving the problem with good accuracy both in the normal and the superfluid regime, for single-particle space sizes adapted to typical nuclear structure calculations.
\end{abstract}

\section{Introduction - Posing the Problem}

In this paper we would like to address the problem of solutions of the nucleonic motion under the influence of the Hamiltonian composed of the mean-field term, $\hat{H}_{m f}$, and the pairing term $\hat{H}_{\text {pair }}$. We assume, as it is often done, that the nuclear pairing can be introduced in two steps. The first one consists in solving the nuclear mean-field problem (without pairing), what allows us to define the single particle spectrum $\left\{\varepsilon_{\alpha}\right\}$ and the corresponding quantum eigenstates $|\alpha\rangle=a_{\alpha}^{+}|0\rangle$, according to the standard notation that uses the particle number representation. Assuming that those mean-field solutions are already known, we may replace the mean-field Hamiltonian by its diagonal representation. The pairing Hamiltonian in its so-called 
"state-dependent form" can be written down as

$$
\hat{H}_{\text {pair }}=-\sum_{\alpha=1}^{\Omega} \sum_{\beta=1}^{\Omega} G_{\alpha \beta} a_{\alpha}^{+} a_{\bar{\alpha}}^{+} a_{\bar{\beta}} a_{\beta} .
$$

Introducing a short-hand notation for the particle-number, $\hat{N}_{\alpha}$, and the pair, $\hat{P}_{\alpha}^{+}$ operators

$$
\hat{N}_{\alpha}=a_{\alpha}^{+} a_{\alpha}+a_{\bar{\alpha}}^{+} a_{\bar{\alpha}} \quad \text { and } \quad \hat{P}_{\alpha}^{+}=a_{\alpha}^{+} a_{\bar{\alpha}}^{+},
$$

we can re-write the Hamiltonian under consideration as follows:

$$
\hat{H}=\hat{H}_{m f}+\hat{H}_{\text {pair }}=\sum_{\alpha=1}^{\Omega}\left(\varepsilon_{\alpha}-\lambda\right) \hat{N}_{\alpha}-\sum_{\alpha, \beta=1}^{\Omega} G_{\alpha \beta} \hat{P}_{\alpha}^{+} \hat{P}_{\beta} .
$$

Most of the calculations and results discussed in this article can be performed using explicitly the state dependent Hamiltonian. However, for the sake of simplicity, especially when comparing our test-results with those of the other authors, we shall restrict ourselves in this article to the constant pairing Hamiltonian; in fact in the corresponding computer codes we replaced $G_{\alpha \beta} \rightarrow G$ for this purpose. Furthermore it is assumed that the single-particle levels are doubly-degenerate, and that $\bar{\alpha}$ represents the conjugated partner of the state $\alpha$ in each doublet. The conjugation symbol "bar" $(\alpha \leftrightarrow \bar{\alpha})$ can be thought of as representing time-reversal conjugation, but also a conjugation according to some other dichotomic symmetries such as signature or simplex.

As the main goal of this article is to compare various algorithms of solution to the Hamiltonian problem introduced above, we will use the so called "picket-fence spectrum" for the single-particle states. Accordingly, the single-particle states are taken to be equidistant $\left(\varepsilon_{\alpha}=\alpha \varepsilon\right.$ with $\left.\varepsilon=1 \mathrm{MeV}\right)$. This allows to standardize the properties of the single-particle spectrum which mimics the presence of some mean-field, but otherwise has no influence on the functioning of the compared algorithms. The exact solution of this problem has been proposed already in the 60 's by Richardson ${ }^{1}$; it is based on the solution of the set of coupled nonlinear equations

$$
\frac{1}{G}+2 \sum_{j=1 ; j \neq i}^{N} \frac{1}{E_{j}-E_{i}}=\sum_{n=1}^{2 N} \frac{1}{2 n-E_{i}}, \quad i=1, \ldots, N
$$

where $N$ represents the number of pairs of particles in the system. The Richardson solutions will serve as the exact test-case for all the approximate algorithms studied in this article.

In what follows we will consider the system as half-filled, and it may therefore be convenient to introduce a parameter ("chemical potential") $\lambda$ according to the following expression:

$$
\lambda=\varepsilon\left(N+\frac{1}{2}\right)-\frac{G}{2},
$$


which will ensure particle-hole symmetry. Further on, by introducing the auxilliary operators for particle $(p)$ and hole $(h)$ states

$$
\hat{M}_{p}=\hat{N}_{p}, \quad \hat{M}_{h}=2-\hat{N}_{h}, \quad \hat{Q}_{p}^{+}=\hat{P}_{p}^{+}, \quad \hat{Q}_{h}=-\hat{P}_{h}^{+}
$$

and by labelling the single-particle energies for particles and holes as

$$
\varepsilon_{p}=\varepsilon(N+p), \quad \varepsilon_{h}=\varepsilon(N-h+1), \quad p, h=1, \ldots, N,
$$

it can easily be shown that the Hamiltonian of Eq. (3) can be expressed in the form (cf. Hirsch et. al. ${ }^{2}$ )

$$
\begin{array}{r}
\hat{H}=-\varepsilon N^{2}+\sum_{p=h=1}^{N}\left[\varepsilon\left(p-\frac{1}{2}\right)+\frac{G}{2}\right]\left(\hat{M}_{p}+\hat{M}_{h}\right) \\
-G \sum_{p p^{\prime}} \hat{Q}_{p}^{+} \hat{Q}_{p^{\prime}}-G \sum_{h h^{\prime}} \hat{Q}_{h}^{+} \hat{Q}_{h^{\prime}} \\
+G \sum_{p h}\left(\hat{Q}_{p}^{+} \hat{Q}_{h}^{+}+\hat{Q}_{p} \hat{Q}_{h}\right)
\end{array}
$$

wherefrom the particle-hole symmetry is obvious.

\section{The SCRPA Approach and its Simplifications}

We recall here briefly the main results of the SCRPA approach to the pairing problem in the case of the picket-fence model, as given by Hirsh et al. ${ }^{2}$.

The two-particle addition and removal operators are defined according to

$$
\hat{A}_{\tau}^{+}=+\sum_{p} X_{p}^{\tau} \hat{\bar{Q}}_{p}^{+}-\sum_{h} Y_{h}^{\tau} \hat{\bar{Q}}_{h}
$$

and

$$
\hat{R}_{\kappa}^{+}=-\sum_{p} Y_{p}^{\kappa} \hat{\bar{Q}}_{p}+\sum_{h} X_{h}^{\kappa} \hat{\bar{Q}}_{h}^{+}
$$

where by definition

$$
\hat{\bar{Q}}_{p}^{+}=\frac{\hat{Q}_{p}^{+}}{\sqrt{\left\langle\hat{D}_{p}\right\rangle}}, \quad \hat{\bar{Q}}_{h}^{+}=\frac{\hat{Q}_{h}^{+}}{\sqrt{\left\langle\hat{D}_{h}\right\rangle}}
$$

In the above expressions we make use of the operators

$$
\hat{D}_{p}=1-\hat{M}_{p}=1-\hat{N}_{p}, \quad \hat{D}_{h}=1-\hat{M}_{h}=-\left(1-\hat{N}_{h}\right)
$$

introduced by Dinh Dang ${ }^{3}$.

The SCRPA equations can now be derived and be brought into the matrix form

$$
\left(\begin{array}{rr}
A & B \\
-B & C
\end{array}\right)\left(\begin{array}{l}
X \\
Y
\end{array}\right)=\hbar \Omega_{\tau}\left(\begin{array}{l}
X \\
Y
\end{array}\right),
$$


where the explicit forms of the sub-matrix blocs are

$$
\left\{\begin{aligned}
A_{p p^{\prime}}^{S C R P A} & =+2\left\{\left[\varepsilon\left(p-\frac{1}{2}\right)+\frac{G}{2}\right]+\frac{G}{\left\langle\hat{D}_{p}\right\rangle}\left[\sum_{p^{\prime \prime}}\left\langle\hat{Q}_{p^{\prime \prime}}^{+} \hat{Q}_{p}\right\rangle-\sum_{h^{\prime \prime}}\left\langle\hat{Q}_{p} \hat{Q}_{h^{\prime \prime}}\right\rangle\right]\right\} \delta_{p p^{\prime}} \\
& -G \frac{\left\langle\hat{D}_{p} \hat{D}_{p^{\prime}}\right\rangle}{\sqrt{\left\langle\hat{D}_{p}\right\rangle\left\langle\hat{D}_{p^{\prime}}\right\rangle}} \\
B_{p h}^{S C R P A} & =G \frac{\left\langle\hat{D}_{p} \hat{D}_{h}\right\rangle}{\sqrt{\left\langle\hat{D}_{p}\right\rangle\left\langle\hat{D}_{h}\right\rangle}} \\
C_{h h^{\prime}}^{S C R P A} & =-2\left\{\left[\varepsilon\left(h-\frac{1}{2}\right)+\frac{G}{2}\right]+\frac{G}{\left\langle\hat{D}_{h}\right\rangle}\left[\sum_{h^{\prime \prime}}\left\langle\hat{Q}_{h}^{+} \hat{Q}_{h^{\prime \prime}}\right\rangle-\sum_{p^{\prime \prime}}\left\langle\hat{Q}_{p^{\prime \prime}}^{+} \hat{Q}_{h}^{+}\right\rangle\right]\right\} \delta_{h h^{\prime}} \\
& +G \frac{\left\langle\hat{D}_{h} \hat{D}_{h^{\prime}}\right\rangle}{\sqrt{\left\langle\hat{D}_{h}\right\rangle\left\langle\hat{D}_{h^{\prime}}\right\rangle}} .
\end{aligned}\right.
$$

By neglecting the mean-values $\left\langle\hat{Q}_{p^{\prime}}^{+} \hat{Q}_{p}\right\rangle,\left\langle\hat{Q}_{p} \hat{Q}_{h}\right\rangle$ and $\left\langle\hat{Q}_{h}^{+} \hat{Q}_{h^{\prime}}\right\rangle$, and by assuming the simplification $\left\langle\hat{D}_{i} \hat{D}_{j}\right\rangle \simeq\left\langle\hat{D}_{i}\right\rangle\left\langle\hat{D}_{j}\right\rangle$, one obtains the standard renormalized RPA (shortly denoted as r-RPA) equations ${ }^{4}$, for which

$$
\left\{\begin{array}{l}
A_{p p^{\prime}}^{r-R P A}=+2\left[\varepsilon\left(p-\frac{1}{2}\right)+\frac{G}{2}\right] \delta_{p p^{\prime}}-G \sqrt{\left\langle\hat{D}_{p}\right\rangle\left\langle\hat{D}_{p^{\prime}}\right\rangle} \\
B_{p h}^{r-R P A}= \\
G \sqrt{\left\langle\hat{D}_{p}\right\rangle\left\langle\hat{D}_{h}\right\rangle} \\
C_{h h^{\prime}}^{r-R P A}=-2\left[\varepsilon\left(h-\frac{1}{2}\right)+\frac{G}{2}\right] \delta_{h h^{\prime}}+G \sqrt{\left\langle\hat{D}_{h}\right\rangle\left\langle\hat{D}_{h^{\prime}}\right\rangle} .
\end{array}\right.
$$

Further on, the standard RPA equations in the particle-particle channel (pp-RPA) can be obtained by setting $\left\langle\hat{D}_{p}\right\rangle=\left\langle\hat{D}_{h}\right\rangle=1$, which leads to

$$
\left\{\begin{array}{l}
A_{p p^{\prime}}^{(p p)-R P A}=+2\left[\varepsilon\left(p-\frac{1}{2}\right)+\frac{G}{2}\right] \delta_{p p^{\prime}}-G \\
B_{p h}^{(p p)-R P A}= \\
C_{h h^{\prime}}^{(p p)-R P A}=-2\left[\varepsilon\left(h-\frac{1}{2}\right)+\frac{G}{2}\right] \delta_{h h^{\prime}}+G .
\end{array}\right.
$$

\section{Particle-Particle Hole-Hole TDA Calculations and Beyond}

A particle-particle Tamm-Dancoff (pp-TDA) state can be written as (see for instance Ring and Schuck $^{5}$, p. 288)

$$
|p p-T D A, \tau\rangle=\sum_{m} C_{m}^{\tau} a_{m}^{+} a_{\bar{m}}^{+}|H F\rangle .
$$

This state is actually described by the (pp-RPA) two-particle operator given in Eq. (9), where one would put the coefficients $Y_{h}^{\tau}$ to zero, when acting on the Hartree-Fock state of the initial system. Based on the same philosophy in which one remarks that the pairing interaction favours pair-excitations, one can construct what we will call a pphh-TDA state, which we express as

$$
|p p h h-T D A, \tau\rangle=\mathcal{C}_{00}^{\tau}|H F\rangle+\sum_{m} \sum_{i} \mathcal{C}_{m i}^{\tau} a_{m}^{+} a_{\bar{m}}^{+} a_{\bar{i}} a_{i}|H F\rangle,
$$


where $|H F\rangle$ represents the Hartree-Fock state of the system, i.e. the state where all the single-particle states below the Fermi level are occupied, whereas all the states above are empty (ground-state of the system where one sets the pairing interaction to zero). By solving directly the associated secular equation one finds

$$
\sum_{n j} H_{m i ; n j} \mathcal{C}_{n j}^{\tau}=E_{\tau} \mathcal{C}_{m i}^{\tau}
$$

where the matrix elements are given by

$$
H_{m i ; n j}=\left\{\sum_{i^{\prime}}\left[2\left(\varepsilon_{i^{\prime}}-\lambda\right)-G_{i^{\prime} i^{\prime}}\right]+2\left(\varepsilon_{m}-\varepsilon_{i}\right)+2 G_{i i}\right\} \delta_{i j} \delta_{m n}-\delta_{i j} G_{m n}-\delta_{m n} G_{i j} .
$$

The advantage of this method is that it gives directly access to states belonging to the initial system, as one excites a pair of particles and holes at the same time. It is clear that, for the addition modes, the pp-TDA method represents a first approximation to the pph-TDA calculations, in which one would restrict oneselves to the subclass of one pair states originating from the level located immediately below the Fermi level for the $(\mathrm{A}+2)$-nucleons system.

The pphh-TDA calculations are based on the excitation of one pair of particles (called 1-pair states) from below to above the Fermi level. This principle can now be generalized to excitations of 2, 3 etc. pairs. Such a generalization has already been proposed some years ago as the PSY-MB method ${ }^{6}$, in which a pre-selection of the many-particle configurations into 1-pair, 2-pairs, 3-pairs... states is performed, together with a given energy cut-off, before the direct diagonalization with the help of the Lanczos procedure (the reader is refered to this latter reference for more details).

\section{System of 24 Particles on 48 Levels}

In the following sections we will focus on two different cases: first we will investigate the results obtained for a system of 24 particles on 48 levels, and than we will concentrate on a larger system composed of 32 particles on 64 levels. Here we begin with the "smaller" one.

\subsection{Ground-state correlation energy}

In this section we investigate the ground-state correlation energy of a system composed of 24 particles distributed over 48 single-particle levels (grouped in 24 doublets). For this system, the total number of seniority zero configurations is equal to 2704156.

In the PSY-MB calculations we retain for the calculations the ground state configuration, all the 144 1-pair states, all the 4356 2-pairs states, and finally all the 484003 -pairs states.

In Fig. 1 are plotted the correlation energy differences between various solutions and the exact one (Richardson), for the ground-state of the 24 particles system, 
in function of the strength of the pairing interaction. In this figure we have also reported the solution obtained within the boson mapping procedure (in its "B" version) proposed by Gambacurta et. al. ${ }^{7}$. From this figure, it is seen clearly that

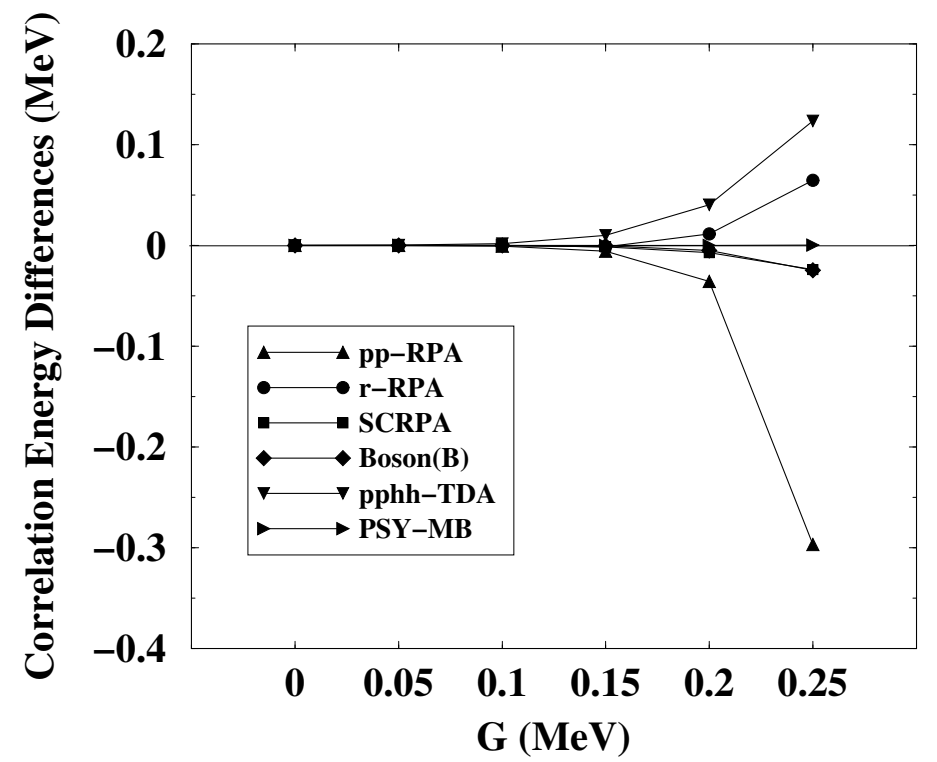

Fig. 1. Correlation energy differences between various approaches and the exact one for the system of 24 particles on 48 single-particle levels, in function of the pairing strength $G$.

there are too strong correlations in the pp-RPA ground-state, especially when one comes close to the "RPA collapse" point. In contrast, they are too weak in the pphh-TDA and r-RPA ground-states. It is also seen that the SCRPA and the Boson formalism ("B" version) give almost identical results (in the Figure, the two curves cannot be distinguished from one another). The striking feature is the fact that the PSY-MB method gives almost exact results, while only $2 \%$ of the total seniority zero basis states are used.

In order to demonstrate that the PSY-MB method provides also good results for the regime of large interaction strengths, we have reported in Fig. 2 the correlation energy for the same system as previously, but for values of $G$ up to $0.54 \mathrm{MeV}$, in the case of the PSY-MB and the pphh-TDA calculations. It is clearly seen that in this regime, the pphh-TDA approximation, which is shown for comparison, provides a poor description of the correlation energy. 


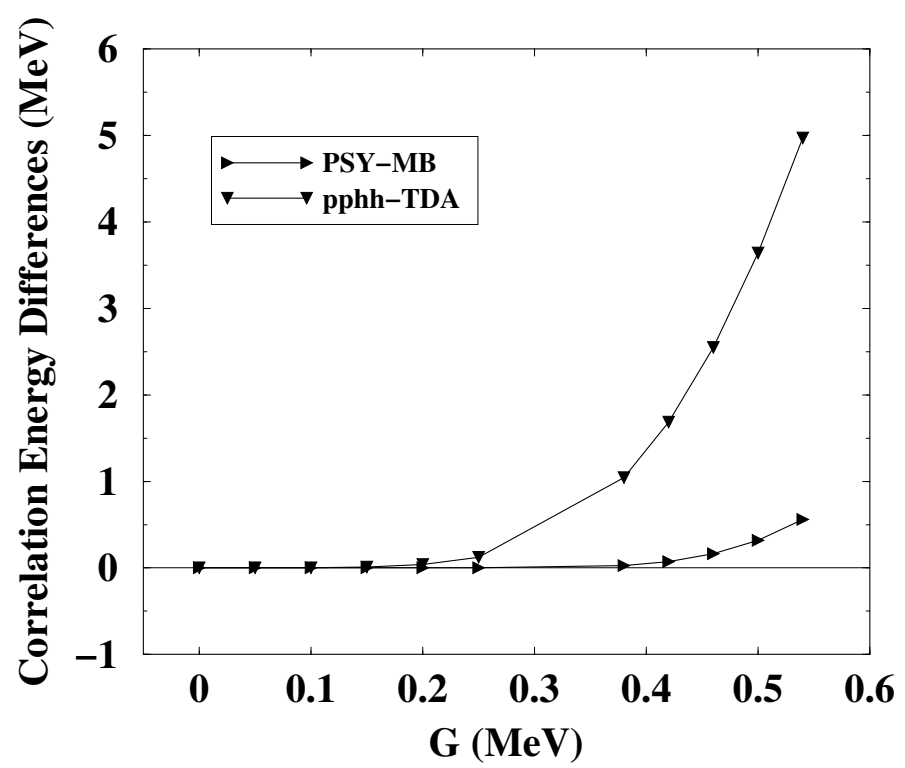

Fig. 2. The same as in Fig. 1, but for the PSY-MB and the pphh-TDA case, also for large values of $G$.

\subsection{The first and the second addition modes}

We can now proceed to calculate the excitation energy of the first and second addition modes, i.e. the lowest energy and the first excitation of a system with 26 particles on 48 equi-spaced levels, with respect to the ground-state energy of the 24 particle system.

It can be seen clearly from Fig. 3, and this has already been pointed out by Hirsch et. al. ${ }^{2}$, that both the pp-RPA and the r-RPA results show the wrong tendency for the first addition mode energy to decrease (it is seen that this is also the case for the pp-TDA calculations). In contrast, the correct trend to increase is given by the SCRPA, the pphh-TDA and the PSY-MB methods. It should be noted that on the figure the results of the pphh-TDA and the PSY-MB calculations are so close that they can hardly be distinguished. It is also interesting to note that the simpler pp-TDA calculations seem to lead to better results than the more refined pp-RPA or r-RPA versions.

As what concerns the second addition mode (see Fig. 4), one notices basically a very similar behaviour of the pp-RPA, the r-RPA and the pp-TDA solutions than for the first addition mode. However, the results are no longer satisfactory for the pphh-TDA case. The important result here is that the SCRPA and the PSY-MB results are extremely close. 
October 25,

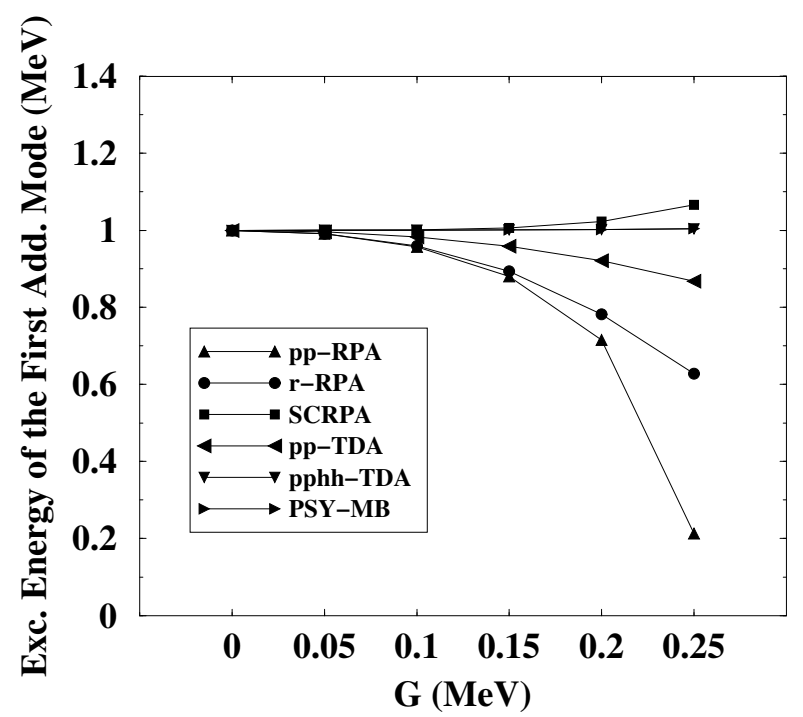

Fig. 3. First addition mode excitation energy for the system of 24 particles on 48 single-particle levels in function of the pairing strength $G$.

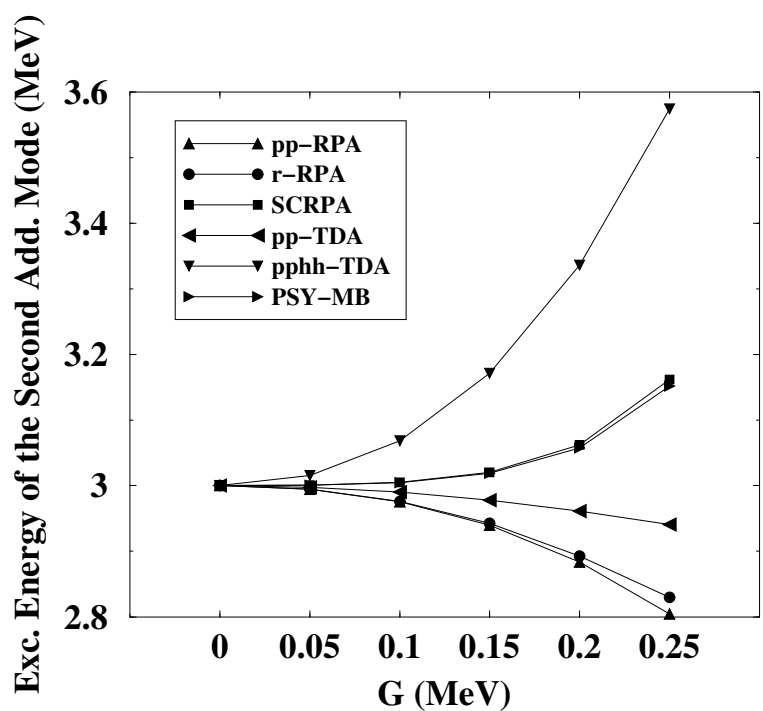

Fig. 4. The same as in Fig. 3, but for the second addition mode excitation energy.

\section{System of 32 Particles on 64 Levels}

In order to allow for realistic calculations valid in the context of typical nuclear structure calculations, the number of single-particle states should be larger, and 
therefore we will now focus on a system composed of 32 particles distributed on 64 single-particle states. In this case, the total number of seniority zero configurations is 601080 390. A simple counting shows furthermore that among these there are 256 1-pair, 14400 2-pairs and 313600 3-pairs configurations. For numerical reasons, we have restricted ourselves, in the PSY-MB case, to the use of all the 1- and 2-pairs states, together with at most 76113 3-pairs states. This means that the results for the PSY-MB calculations are obtained with the use of less than $0.02 \%$ of the seniority zero configurations.

\subsection{Ground state and first excitation energy}

In Figs. 5 and 6 we show the results for the calculations of the correlation energy of the ground state, as well as the first excitation energy. As we would like to study

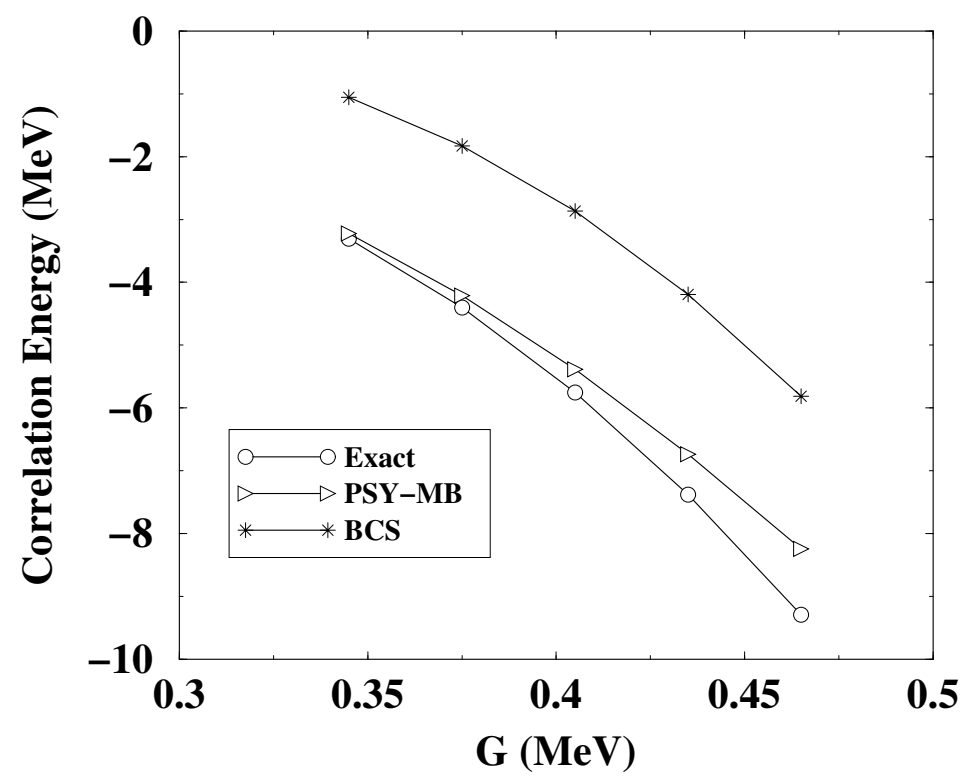

Fig. 5. Ground-state correlation energy for the system of 32 particles on 64 single-particle levels in function of the pairing strength $G$.

here in more detail the superfluid regime, we perform a direct comparison with the standard BCS results. It is clear that the PSY-MB calculations give again a good agreement with the exact results, in both cases, whereas the BCS calculations show globally an overestimation of both quantities. In the two cases, one observes that the results diverge more from the exact ones, as the pairing interaction strength parameter $G$ increases. 


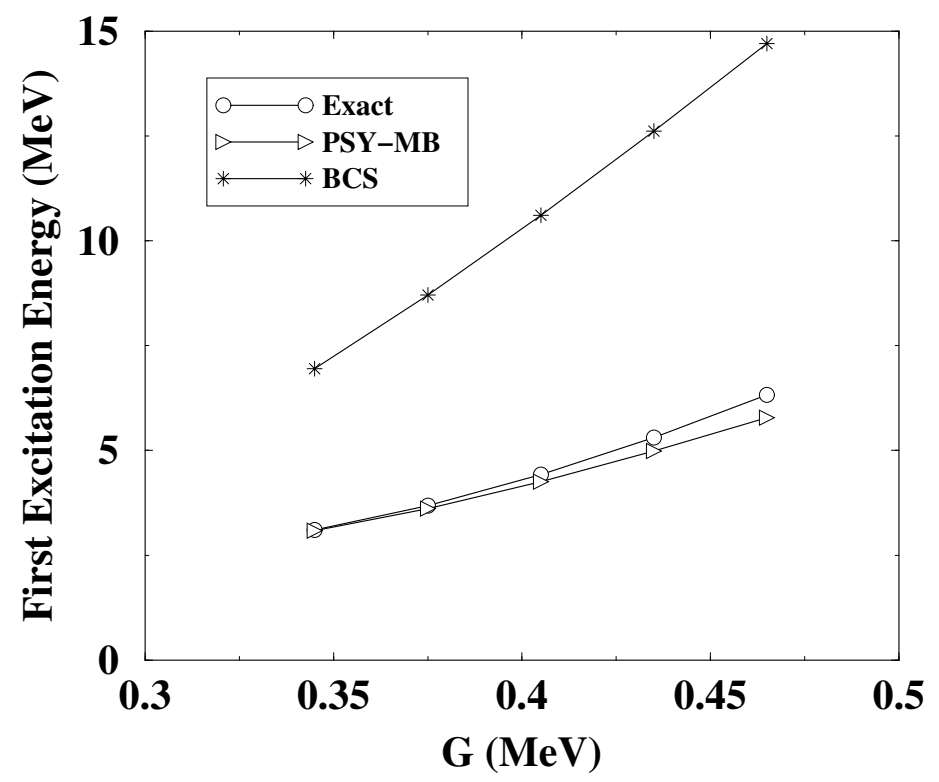

Fig. 6. Similar as in Fig. 5, but for the first excitation energy.

\subsection{Single-particle occupation probabilities}

In Fig. 7 and 8 are shown the occupation probabilities for the nearest single-particle levels around the Fermi level, for the system of 32 particles on 64 states, for two different values of the pairing interaction parameter $G$.

It is seen that in the case of the PSY-MB calculations, the results are better for the lower value of the pairing interaction, which is in accordance with the conclusions given for the ground-state correlation energy and the first excitation energy. However, the situation is different in the BCS case, where one notices that the occupation probabilities are in better agreement with the exact calculations for the larger value of $G$.

\section{Conclusions}

In this article we have shown that the so-called PSY-MB method introduced already some years ago provides a very powerful tool for solving the problem of the pairing hamiltonian in realistic situations occuring in the context of nuclear physics.

As an illustration we have compared our results with several variants of the Random Phase Approximation that is widely used in the litterature, also in its sophisticated fully self-consistent version. It has been shown that a high degree of precision can be achieved within the framework of the PSY-MB calculations, both in the normal and in the superfluid regime, treated in one single formalism. In particular, no abrupt phase transition is seen, as it should be the case for the 
October 25,

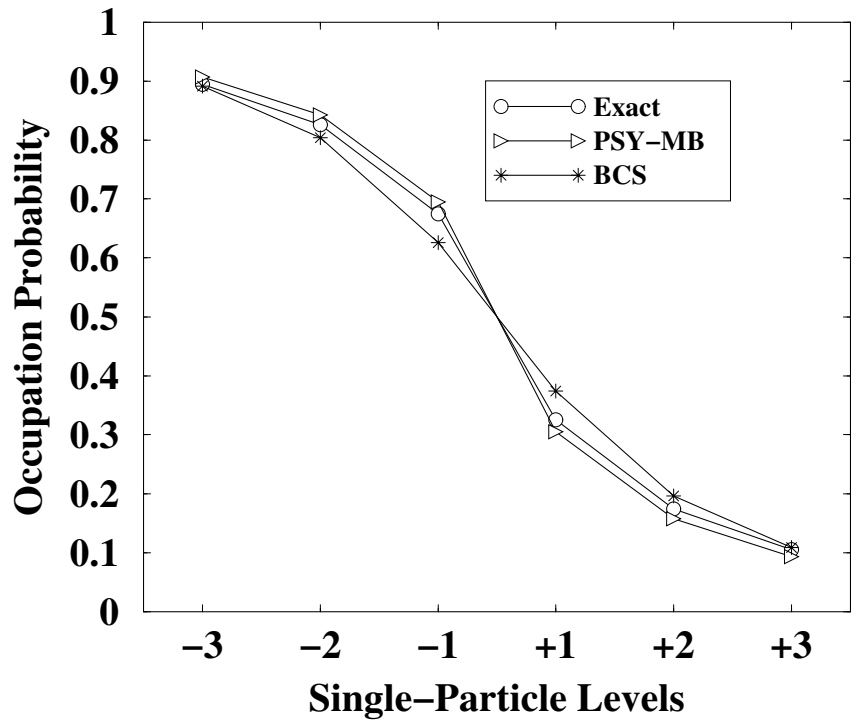

Fig. 7. Single-particle occupation probabilities for the three doublets below and above the Fermi level, in the ground-state solution, for the system of 32 particles on 64 single-particle levels, with $G=0.375 \mathrm{MeV}$.

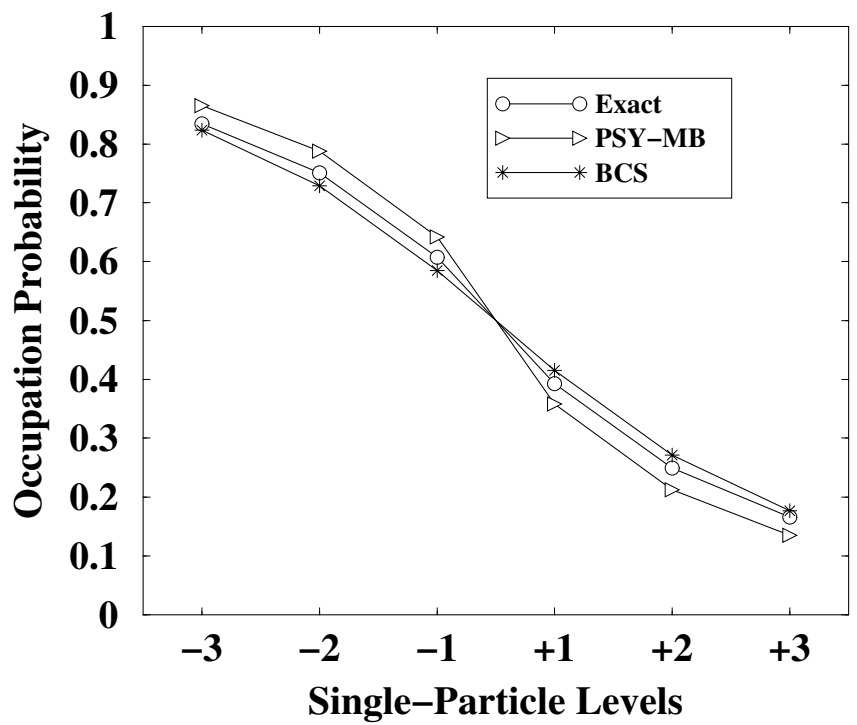

Fig. 8. Similar as in Fig. 7, but for $G=0.435 \mathrm{MeV}$. 
October 25,

molique dudek`200605

finite systems. The results are reliable both from the point of view of the correlation energies, but also the excitation energies, as well as the single-particle occupation probabilities.

In this paper a model space for the equidistant single-particle spectrum has been used (the so-called "picket-fence model"), allowing for a direct comparison with the exact results given by Richardson. However, the PSY-MB method can be applied without no change to any physical situation where the single-particle energies would be provided by some potential of the Woods-Saxon or Hartree-Fock type for instance. An important advantage of the method lies in the fact that not only seniority zero solutions can be obtained. Higher seniority cases are even treated with more ease, since the corresponding many-body configuration spaces are smaller due to the blocking effect.

\section{References}

1. R.W. Richardson, Phys. Rev. 141, 949 (1966).

2. J.G. Hirsh, A. Mariano, J. Dukelsky and P. Schuck, Ann. Phys. 296, 187 (2002).

3. N. Dinh Dang, Phys. Rev. C71, 024302 (2005).

4. D.J. Rowe, Phys. Rev. 175, 1283 (1968).

5. P. Ring and P. Schuck The Nuclear Many-Body Problem, Springer-Verlag, New-York, Heidelberg, Berlin, 1980.

6. H. Molique and J. Dudek, Phys. Rev. C56, 1795 (1997).

7. D. Gambacurta, M. Sambatoro and F. Catara, Phys. Rev. C73, 014310 (2006). 